\title{
Frequency Based Analysis of Voting Rules
}

Citation for published version (APA):

Chatterjee, S., \& Storcken, T. (2017). Frequency Based Analysis of Voting Rules. Maastricht University, Graduate School of Business and Economics. GSBE Research Memoranda No. 006 https://doi.org/10.26481/umagsb.2017006

Document status and date:

Published: 23/03/2017

DOI:

10.26481/umagsb.2017006

Document Version:

Publisher's PDF, also known as Version of record

\section{Please check the document version of this publication:}

- A submitted manuscript is the version of the article upon submission and before peer-review. There can be important differences between the submitted version and the official published version of record.

People interested in the research are advised to contact the author for the final version of the publication, or visit the DOI to the publisher's website.

- The final author version and the galley proof are versions of the publication after peer review.

- The final published version features the final layout of the paper including the volume, issue and page numbers.

Link to publication

\footnotetext{
General rights rights.

- You may freely distribute the URL identifying the publication in the public portal. please follow below link for the End User Agreement:

www.umlib.nl/taverne-license

Take down policy

If you believe that this document breaches copyright please contact us at:

repository@maastrichtuniversity.nl

providing details and we will investigate your claim.
}

Copyright and moral rights for the publications made accessible in the public portal are retained by the authors and/or other copyright owners and it is a condition of accessing publications that users recognise and abide by the legal requirements associated with these

- Users may download and print one copy of any publication from the public portal for the purpose of private study or research.

- You may not further distribute the material or use it for any profit-making activity or commercial gain

If the publication is distributed under the terms of Article $25 \mathrm{fa}$ of the Dutch Copyright Act, indicated by the "Taverne" license above, 


\section{Maastricht University}

Swarnendu Chatterjee,

Ton Storcken

Frequency Based Analysis of Voting Rules

RM/17/006

\section{GSBE}

Maastricht University School of Business and Economics

Graduate School of Business and Economics

P.O Box 616

NL- 6200 MD Maastricht

The Netherlands 


\title{
Frequency Based Analysis of Voting Rules
}

\author{
Swarnendu Chatterjee · Ton Storcken
}

March 21, 2017

\begin{abstract}
In a large electorate it is natural to consider voters' preference profiles as frequency distributions over the set of all possible preferences. We assume coherence in voters' preferences resulting in accumulation of voters preferences. We show that such distributions can be studied via superpositions of simpler so called unimodal distributions. At these, it is shown that all well-known rules choose the mode as the outcome. We provide a set of sufficient conditions for a rule to have this trait of choosing the mode under unimodal distributions. Further we show that Condorcet consistent rules, Borda rule, plurality rule are robust under tail-perturbations of unimodal distributions.
\end{abstract}

Keywords Voting · Unimodal distribution · Condorcet consistent rule · Borda Rule · Plurality rule JEL Classification D71, D72

\section{Introduction}

We focus on voting situations involving large numbers of voters like nation wide elections. In these voting situations voters' preferences over the candidates may vary considerably. On the other hand it is reasonable to expect that cultural backgrounds, social media, individual networks etc. induce some coherence between these voters' preferences. Coherence expressed in one or several accumulations of voters' preferences. Representing the voters' preference combinations, or profiles, at such large electorates by frequency distributions each accumulation can be seen as an agglomeration of preferences around a local mode. In that way the whole profile can be viewed as the addition or superposition of such (local) unimodal distributions yielding a multimodal distribution. In this paper we study unimodal distributions and how reasonable outcomes of these are related to reasonable outcomes at multimodal distributions.

Taking the set of linear orders over all candidates as the admissible set of preferences a profile is a frequency distribution on this set of linear orders. This set is structured by the Kemeny distance counting the number of discordant pairs as the distance between two such orders. This distance is the minimal path-length needed to convert the one order into the other by swapping consecutively ordered candidates.

Swarnendu Chatterjee

Department of Quantitative Economics, School of Business and Economics, Maastricht University

Tel.: +31617509371

E-mail: s.chatterjee@maastrichtuniversity.nl

Ton Storcken

Department of Quantitative Economics, School of Business and Economics, Maastricht University Tel.: +31433883728

E-mail: t.storcken@maastrichtuniversity.nl 
Starting from one order at each step we arrive at a new order which has one discordant pair less with the final order than the previous order on that path. A unimodal distribution is a distribution where one order, the mode, has highest frequency and where further frequency decreases with the distance (in Kemeny sense) from the mode. Multimodal distributions are considered to be additions of such unimodal distribution. It is very reasonable to choose the mode at unimodal distribution as many well-known choice rules do so. Likewise when analysing multimodal distributions it appears natural to infer the outcome from the intersection of the local modes. This opens the possibility to study such more general distributions via unimodal ones.

We start with observing the behaviour of several well-known rules like Condorcet consistent rules, Scoring rules, Elimination rules, Kemeny-like rules, under unimodal distributions. It is worth noting that all these rules choose the mode as the outcome under unimodal distribution. We also provide a set of sufficient conditions which ensures that any rule satisfying them choose the mode at a unimodal distribution. To investigate the robustness of this result we allow for disturbances in the tail part of a unimodal distribution and show that even under this tail-perturbed distributions Condorcet consistent rules, Borda rule and plurality rule chooses the mode as the outcome.

Intuitively, for many rules the property of choosing the mode follows because of the following. Let at the mode candidate $a$ be preferred to candidate $b$. Then a preference $R$ at which $a$ is preferred to $b$ is closer to the mode than the corresponding preference $R^{\prime}$ at which the positions of $a$ and $b$ in $R$ are swapped. Therefore $R$ has higher frequency than $R^{\prime}$. Letting $R$ run over the set of linear orders where $a$ is preferred to $b$ lets consequently $R^{\prime}$ run over the complementary set. So, in pairwise comparison $a$ will beat $b$ and the mode consists of all pairs which in pairwise comparisons beat each other: the Condorcet order. The same argument of $R$ having higher frequency than $R^{\prime}$ implies that at any score rule $a$ gets a higher score than $b$ and that $b$ is eliminated before $a$ is. So, the mode also embodies the "positional" order as well as the order of "elimination". Formalising this intuition we get some sufficient conditions. Besides anonymity and neutrality, there are two important conditions that are sufficient for this property of choosing the mode, namely, discrimination and monotonicity. Here anonymity and neutrality are used in their usual sense. Monotonicity and discrimination are formally defined in the following section. In general monotonicity properties are restrictive and therefore not satisfied by reasonable collective decision rules. The one discussed here is satisfied by many rules, for instance pairwise rules and score rules. It demands that the pair $a b$ is in the outcome at profile $q$ if it is in the outcome at profile $p$, whenever $q$ is obtained from $p$ by changing only the preference of some voters with a preference like $R^{\prime}$ at which $b$ is preferred to $a$ to a preference $R$ obtained by swapping the position of $a$ and $b$ in $R^{\prime}$. In this way monotonicity is defined pairwise but it is sensitive to the positions of candidates. It is therefore satisfied by many rules. Discrimination means, that a rule cannot be indifferent between two candidates, say $a$ and $b$, whenever frequency of every preference $R$ at which $a$ is preferred to $b$ out numbers frequency of $R^{\prime}$. Here $R^{\prime}$ is the preference obtained by swapping the position of $a$ and $b$ in $R$.

In the next part, for Condorcet consistent rules, Borda rule and plurality rule we show that, all three assign the mode at the following type of perturbed unimodal distribution. Frequency declines with the distance to the mode until about half the maximal distance ${ }^{m}$. Beyond this point frequencies are free but constant at each specific distance from the mode and they are bounded above by the frequency at half the maximal distance. The combinatorial reasoning by which these results are deduced might work similarly for other collective decision rules such as other score rules or some elimination rules. But we have not found a general method to deduce this, for instance, for all score rules. The result found here indicate, however, that the mode choice of Condorcet consistent rules, Borda rule and plurality rule are robust to considerable perturbations in the tail part of a single mode distribution. For a multimodal distribution, we show that the outcomes at each of the unimodal constituents together determine the outcome of the original distribution. For instance, fordiscriminating collective decision rules we show, that the outcome

\footnotetext{
1 Taking $m$ the number of candidates maximally $\left(\begin{array}{c}m \\ 2\end{array}\right)$ pairs can be discordant.
} 
at the union of two unimodal distributions is between the two modes of these unimodal distributions. This result generalises to superpositions of more than two unimodal distributions. However, it is evident that at these distributions, particularly when the modes have an empty intersection, we might end up in difficult situations like Condorcet profiles. A deeper study of these situations is certainly necessary, but beyond the scope of this first exploration of unimodal distributions.

There have been some empirical work with the so-called impartial culture or uniform distribution, where each preference order is possible and occurs with equal probability. Under this assumption, the probability of a majority cycle occurring has been calculated (see Gehrlein (2006) [?], Riker (1982, p. 122)[?]. Many authors have noted that the impartial culture is a significant idealisation that almost certainly does not occur in real-life elections. Grofman et al. (2003)[?] go even further arguing that the impartial culture is a worst-case scenario in the sense that any deviation results in lower probabilities of a majority cycle (see Grofman et al. (2006)[?], for a complete discussion of this issue). A few other probabilistic models have been developed, for instance, Multinomial likelihood models (Gillett, 1976[?], 1978[?]), Dual Culture (Gehrlein; 1978)[?], Maximal Culture Condition (Fishburn and Gehrlein, 1977 [?]). All these indicate that when voters' preferences are homogeneous, there is a increased likelihood that a Pairwise Majority Rule Winner exists meaning that Condorcet cycles are avoided. Our result go further on this as at unimodal distributions not only pairwise majority cycles are absent, but also all well-know rules have the same outcome: the mode. The main difference in our approach is that the distributions are structured by the Kemeny distance. A structure that is naturally embedded with the preferences. Merlin et. al. (2004)[?] have studied the probability of conflicts in a U.S. presidential type of election. Their results partially resemble ours, but strictly speaking are not comparable, because of the assumption of impartial culture.

In section ?? we formally introduce the model. Section ?? shows that at unimodal distributions many collective decision rules select the mode as an outcome. In section ?? we formulate some sufficiency conditions for abstract collective decision rules to have the mode as an outcome at such distributions. Section ?? discusses disturbances in the "tail" of unimodal distributions. Section ?? is on multimodal distributions. A discussion in section ?? concludes this paper. To make the paper self contained we provided an appendix section ?? on combinatorial results needed in this paper.

\section{Model}

We consider a finite but large set of voters $N=\{1, \ldots, n\}$, and a finite set of candidates $A=\left\{a_{1}, \ldots, a_{m}\right\}$. We assume that there are at least three candidates, hence $m \geq 3$.

Let $R$ denote a relation on $A$, i.e. $R \subseteq A \times A$. As usual for candidates $x$ and $y,(x, y) \in R$ means that at preference (relation) $R$, candidate $x$ is (weakly) ${ }^{\square}$ preferred to candidate $y$. Individual preferences of voters are formalized here by linear orders, i.e. complete, antisymmetric and transitive relations on $A$. The set of these linear orders on $A$ is denoted by $\mathbb{L}$. Let $-R$ denote the reverse of $R$, i.e. $-R=\{(x, y):(y, x) \in R\}$. For candidates $x$ and $y$ let $\tau_{x y}$ denote the permutation on $A$ defined $\tau_{x y}(z)=z$ if $z \notin\{x, y\}, \tau_{x y}(x)=y$ and $\tau_{x y}(y)=x$. Now $\tau_{x y} R$ denotes the preference relation where the positions of $x$ and $y$ are swapped, i.e. $\tau_{x y} R=\left\{\left(\tau_{x y}(v), \tau_{x y}(w)\right):(v, w) \in R\right\}$.

A profile $p$ (of individual preferences) assigns to every voter $i$ a linear order $p(i)$ in $\mathbb{L}$. Let $\mathbb{L}^{N}$ denote the set of all these profiles, that is the set of all the combinations of individual preferences. To exclude discussions on resolving ties in the collective orders we allow the collective outcomes to be weak orders, i.e. complete and transitive orders on $A$. The set of all these weak orders is denoted by $\mathbb{W}$. Further, $\tau_{x y} p$ is the coordinate-wise extension of $\tau_{x y}$. So, $\left(\tau_{x y} p\right)(i)=\tau_{x y}(p(i))$. For non-empty subsets of voters $S$ profiles $q$ and $p$ are called $S$-deviations if $p(i)=q(i)$ for all $i \in N-S$. Further, $\left.p\right|_{S}$ denotes the profile $p$ restricted to $S$.

\footnotetext{
${ }^{2}$ If $x=y$, then $x$ and $y$ are indifferent. If $x \neq y$, then $x$ is strictly preferred to $y$.
} 
For candidates $x$ and $y$ let $\mathbb{L}_{x y}$ denote the set of linear orders $R$ at which $x$ is strictly preferred to $y$, that is $\mathbb{L}_{x y}=\{R \in \mathbb{L} \mid(x, y) \in R\}$.

The collective decision is formalized by a (preference) rule $e^{[\mathrm{l}}$. From now onwards we use just rule instead of preference rule. $F$, a function that assigns to every profile $p$ in $\mathbb{L}^{N}$ a collective preference $F(p)$ in $\mathbb{W}$.

\subsection{Unimodal Frequency Distributions}

A frequency distribution is formalized by a function representing the number of times each linear order appear in a profile. Given a profile $p$ of individual preferences of the voters and a preference $R$ in $\mathbb{L}$, then $f(R, p)$ denotes the number of voters with preference $R$ at profile $p$, that is

$$
f(R, p)=|\{i \in N \mid p(i)=R\}|,
$$

where $|S|$ denotes the cardinality of an arbitrary set $S$. There is a metric space over $\mathbb{L}$ induced by the Kemeny distance function $d$ defined for two preferences $R^{1}$ and $R^{2}$ in $\mathbb{L}$ as follows ${ }^{\text {ti }}$

$$
d\left(R^{1}, R^{2}\right)=\frac{1}{2}\left|\left(R^{1} \triangle R^{2}\right)\right|
$$

where $\triangle$ denotes the symmetric difference between sets, i.e. $R^{1} \triangle R^{2}=\left(R^{1}-R^{2}\right) \cup\left(R^{2}-R^{1}\right)$. The Kemeny distance indicates the number of discordant pairs.

A profile $p$ is called unimodal if there exists a preference $\widehat{R}$, the mode, such that for every two preferences $R^{1}$ and $R^{2}$ in $\mathbb{L}$, such that $d\left(\widehat{R}, R^{1}\right)<d\left(\widehat{R}, R^{2}\right)$, then we have $f\left(R^{1}, p\right)>f\left(R^{2}, p\right)$.

So, a profile $p$ is unimodal if there is a preference $\widehat{R}$ in $\mathbb{L}$ with highest frequency, such that frequencies for all other preferences in $\mathbb{L}$ strictly decrease in their distance from this mode $\widehat{R}$. A unimodal distribution is called symmetrical if in addition $f\left(R^{1}, p\right)=f\left(R^{2}, p\right)$ whenever $d\left(\widehat{R}, R^{1}\right)=d\left(\widehat{R}, R^{2}\right)$.

Example 1 Example of a symmetric unimodal distribution with 4 candidates $a, b, c, d$.

\footnotetext{
3 Preference rules are usually known as Social Welfare Functions. As the model discussed here also applies to non-welfare issues such as voting we rather use the term preference rule.

4 Here we actually take half the Kemeny distance because between two linear orderings it is a multiple of two.
} 

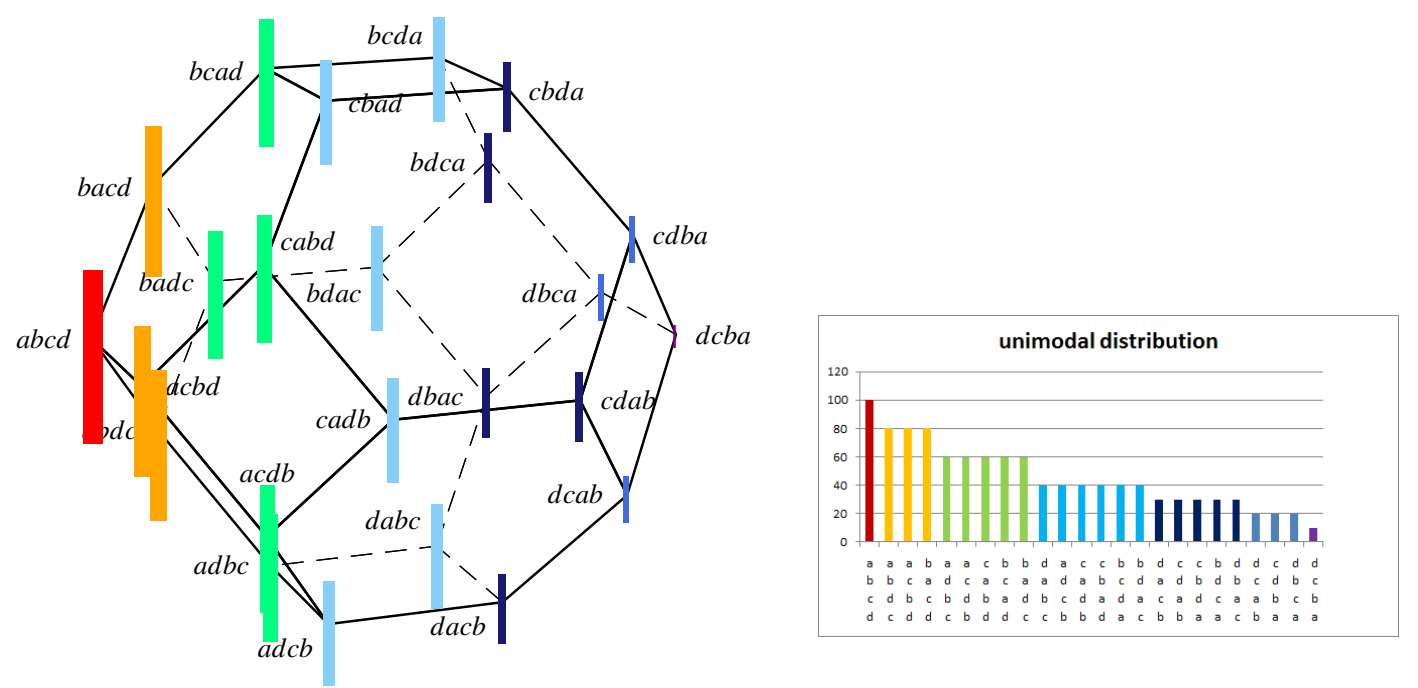

Fig. 1: Symmetric unimodal distribution with 4 candidates

For 4 candidates we have 4 ! = 24 many linear orders in the set $\mathbb{L}$. This set $\mathbb{L}$ can be structured by the Kemeny distance as is shown in the above figure ??: the truncated octahedron. In this structure, an edge between two linear orders means that these have Kemeny distance 1, i.e. they differ by only one swap of consecutively ordered candidates. For instance, take $a b c d$ and bacd. Now we have a frequency distribution on this structure with mode at $a b c d$. The frequency of $a b c d$ is indicated by the length of the red bar. Going one step (in the Kemeny sense) away from $a b c d$, we have three linear orders, namely, $b a c d, a c b d, a b d c$. Their frequencies are lesser compared the mode. For this example, we have taken equal frequencies, so the distribution is symmetric. Their frequencies are indicated by the length of the yellow bars. Similarly we have declining frequencies for the linear orders at distances 2,3,4,5 and 6 from the mode $a b c d$. This same distribution can be viewed as a flat bar chart, where we use the same colors to indicate that those linear orders are at the same distance from the mode. Of course a lot of information is lot transferring from the Kemeny structure to 2 dimensional bar chart.

\subsection{Definitions of Some Conditions}

Preference rules will be discussed with respect to the following conditions.

Anonymity means that the rule is symmetric in its arguments. Anonymity guarantees that every voters' preference is equally important in determining the outcome. The outcome is based on how many times a preference is announced at a certain profile instead of who announced that preference. Let $\sigma: N \rightarrow N$ be a permutation on $N$ the set of voters. Let $p$ be a profile in $\mathbb{L}^{N}$. Then profile $q=p \circ \sigma$ is defined for all voters $i$ by

$$
q(i)=p(\sigma(i))
$$

Rule $F$ is anonymous if for all profiles $p$ and all permutations $\sigma$

\footnotetext{
5 Note that we consider a profile to be a function from $N$ to $\mathbb{L}$.
} 


$$
F(p)=F(p \circ \sigma) .
$$

Neutrality means that the rule treats candidates in equal situations equally. Let $x$ and $y$ be candidates.

Let $\tau_{x y}$ be a permutation on $A$, where it swaps the positions of $x, y \in A$. Rule $F$ is neutral ${ }^{6}$ if for all candidates $x$ and $y$, all profiles $p$ and all $\tau_{x y}$ on $A$

$$
F\left(\tau_{x y} p\right)=\tau_{x y} F(p) .
$$

Monotonicity means that if in comparison of profiles $p$ and $q$ the preference " $x$ is preferred to $y$ " among the voters increases when going from $p$ to $q$, then this preference at the outcomes should not decrease.

Rule $F$ is monotone if for all candidates $x$ and $y$, all non-empty subsets $S$ of $N$ and all $S$-deviations $p$ and $q$, such that both $q(i)=\tau_{x y} p(i)$ and $(y, x) \in p(i)$ for all $i \in S(y, x) \notin F(p)$ implies $(y, x) \notin F(q)$.

Discrimination means that for candidates $x$ and $y$ at a profile $p$ the outcome $F(p)$ is indifferent between these two only if there are $R^{1}$ at which $x$ is strictly preferred to $y$ and $R^{2}$ at which $y$ is strictly preferred to $x$ such that $f\left(R^{t}, p\right) \geqslant f\left(\tau_{x y} R^{t}, p\right)$ for both $t \in\{1,2\}$. So, indifference between $x$ and $y$ in an outcome can only occur if neither all for all $R \in \mathbb{L}_{x y}$ frequencies $f(R, p)$ are strictly greater than $f\left(\tau_{x y} R, p\right)$ nor for all $R \in \mathbb{L}_{y x}$ frequencies $f(R, p)$ are strictly greater than $f\left(\tau_{x y} R, p\right)$.

Rule $F$ discriminates if for all profiles $p$ and all different candidates $x$ and $y$

$(x, y) \in F(p)$ and $(y, x) \in F(p)$ implies, that there are preferences $R^{1} \in \mathbb{L}_{x y}$ and $R^{2} \in \mathbb{L}_{y x}$ with $f\left(R^{t}, p\right) \geqslant f\left(\tau_{x y} R^{t}, p\right)$ for all both $t \in\{1,2\}$.

It should be clear, that one equality between frequencies $f(R, p)$ and $f\left(\tau_{x y} R, p\right)$ for some linear order is sufficient to allow for an indifference outcome between $x$ and $y$ at a discriminating rule. This is also allowed in cases where $f\left(R^{1}, p\right)>f\left(\tau_{x y} R^{1}, p\right)$ and $f\left(R^{2}, p\right)>f\left(\tau_{x y} R^{2}, p\right)$ for some $R^{1} \in \mathbb{L}_{x y}$ and some $R^{2} \in \mathbb{L}_{y x}$. But in cases where $f(R, p)>f\left(\tau_{x y} R, p\right)$ for all $R \in \mathbb{L}_{x y}$ discrimination implies the preference between $x$ and $y$ is strict. It is natural to go one step further in those cases and to impose, that in those situations $x$ should be strictly preferred to $y$. This condition is referred to as positive discrimination.

So, rule $F$ positively discriminates if for all profiles $p$ and all different candidates $x$ and $y(x, y) \in F(p)$ and $(y, x) \notin F(p)$ whenever $f(R, p)>f\left(\tau_{x y} R, p\right)$ for all $R \in \mathbb{L}_{x y}$.

As we frequently use the terms Condorcet consistent rules and pairwise rules in the following sections, let us clarify what we mean by them. Firstly a pairwise comparison matrix is a square matrix with its $(i, j)^{t h}$ element as the number of voters preferring the $i^{\text {th }}$ candidate to the $j^{\text {th }}$ candidate, and all the diagonal elements are zero. Pairwise rules are the rules that provide the same outcome for two different preference profiles as long as the pairwise comparison matrix resulting from these two preference profiles are the same. A Condorcet consistent choice rule chooses the Condorcet winner if it exists. In case of a Condorcet consistent preference rule, the Condorcet order is chosen if it exists. A pairwise rule may not be Condorcet consistent, for example, Borda rule is pairwise but it is not Condorcet consistent. It is not difficult to see, that score rules as well as Condorcet consistent rules or pairwise rules are positively discriminating and monotone. The proofs for this are essentially given in Examples ?? and ?? in Section ??.

\section{Decisions in Unimodal Frequency Distributions}

The following examples show that, at unimodal distributions the mode is the outcome of several wellknown decision rules like score rules, Condorcet consistent or pairwise rules and the Coombs rule. There are many more rules that have the mode as outcome at these frequency distributions. Instead of proving

\footnotetext{
6 The neutrality notion defined here is equivalent to the usual one, because every permutation can be decomposed into pairwise swaps like $\tau_{x y}$.
} 
this we formulate sufficient conditions for an arbitrary rule to assign the mode at such frequency distributions. In all these examples let $\widehat{p}$ be a unimodal profile with mode $\widehat{R}$. The result of these examples are based on the following Lemma.

Lemma 1 Let $x$ and $y$ be two different preferences and $R^{1}, R^{2}$ be two linear orders, such that $(x, y) \in$ $R^{1}$ and $(y, x) \in R^{2}$. Then $d\left(R^{1}, \tau_{x y} R^{2}\right)<d\left(R^{1}, R^{2}\right)$.

Proof We partition $A \backslash\{x, y\}$ as follows:

$$
\begin{aligned}
& B_{1}=\left\{a \in A:(a, x) \in R^{1} \text { and }(a, y) \in R^{2}\right\}, \\
& B_{2}=\left\{a \in A:(a, x) \in R^{1} \text { and }(a, x),(y, a) \in R^{2}\right\}, \\
& B_{3}=\left\{a \in A:(a, x) \in R^{1} \text { and }(x, a) \in R^{2}\right\}, \\
& M_{1}=\left\{a \in A:(x, a),(a, y) \in R^{1} \text { and }(a, y) \in R^{2}\right\}, \\
& M_{2}=\left\{a \in A:(x, a),(a, y) \in R^{1} \text { and }(a, x),(y, a) \in R^{2}\right\}, \\
& M_{3}=\left\{a \in A:(x, a),(a, y) \in R^{1} \text { and }(x, a) \in R^{2}\right\}, \\
& W_{1}=\left\{a \in A:(y, a) \in R^{1} \text { and }(a, y) \in R^{2}\right\}, \\
& W_{2}=\left\{a \in A:(y, a) \in R^{1} \text { and }(a, x),(y, a) \in R^{2}\right\} \text { and } \\
& W_{3}=\left\{a \in A:(y, a) \in R^{1} \text { and }(x, a) \in R^{2}\right\} .
\end{aligned}
$$

The following pictures the preferences $R^{1}, R^{2}$ and $\tau_{x y} R^{2}$.

$$
\begin{aligned}
& B_{1} \cup B_{2} \cup B_{3} \quad x M_{1} \cup M_{2} \cup M_{3} y W_{1} \cup W_{2} \cup W_{3}: R^{1} \\
& B_{1} \cup M_{1} \cup W_{1} \text { y } B_{2} \cup M_{2} \cup W_{2} x B_{3} \cup M_{3} \cup W_{3}: R^{2} \\
& B_{1} \cup M_{1} \cup W_{1} x B_{2} \cup M_{2} \cup W_{2} \text { y } B_{3} \cup M_{3} \cup W_{3}: \tau_{x y} R^{2}
\end{aligned}
$$

Now

$$
\begin{aligned}
d\left(R^{1}, R^{2}\right)= & \frac{1}{2}\left|\left[\left(R^{1} \triangle R^{2}\right) \cap(A-\{x, y\})^{2}\right]\right|+\left|B_{2}\right|+2 \cdot\left|B_{3}\right|+ \\
& \left|M_{1}\right|+2 \cdot\left|M_{2}\right|+\left|M_{3}\right|+2 \cdot\left|W_{1}\right|+\left|W_{2}\right|+1 \\
= & d\left(R^{1}, \tau_{x y} R^{2}\right)+2 \cdot\left|M_{2}\right|+1 \\
> & d\left(R^{1}, \tau_{x y} R^{2}\right)
\end{aligned}
$$

which completes the proof.

Lemma 2 Let $x, y \in A$, with $(y, x) \notin \widehat{R}$ and let $R \in \mathbb{L}_{x y}$. Then $f(R, \widehat{p})>f\left(\tau_{x y} R, \widehat{p}\right)$.

Proof By Lemma ?? it follows that $d(\widehat{R}, R)<d\left(\widehat{R}, \tau_{x y} R\right)$ which easily yields the desired inequality by the unimodality of $\widehat{p}$.

\section{Example 2 Condorcet consistent Rules}

Condorcet consistent rules depend on pairwise majority comparisons of the candidates. In general these comparisons may yield cycles and rules therefore may differ in these situations: different Condorcet consistent rules break up cycles in different ways. If, however, at a certain profile pairwise majority comparisons yield a complete, strict and transitive order from overall winner (the Condorcet winner) to overall loser (the Condorcet loser), then this is the outcome of all these rules at that profile. We argue that at profile $\widehat{p}$ pairwise majority comparisons yield the mode $\widehat{R}$ as the Condorcet order. Herewith we show 
that all Condorcet consistent preference rules assign the mode at a unimodal profile. Let $(x, y) \in \widehat{R}$, with $x \neq y$. It is sufficient to show that

$$
|\{i \in N:(x, y) \in \widehat{p}(i)\}|>|\{i \in N:(y, x) \in \widehat{p}(i)\}| .
$$

Lemma ?? yields that $f(R, \widehat{p})>f\left(\tau_{x y} R, \widehat{p}\right)$ for all $R \in L_{x y}$. Since, $|\{i \in N:(x, y) \in \widehat{p}(i)\}|=\sum_{R \in \mathbb{L}_{x y}} f(R, \widehat{p})$ and $|\{i \in N:(y, x) \in \widehat{p}(i)\}|=\sum_{R \in \mathbb{L}_{x y}} f\left(\tau_{x y} R, \widehat{p}\right)$ the desired inequality follows.

\section{Example 3 Score Rules}

In score rules voters assign scores $s_{1}, s_{2}, \ldots, s_{m}$ to the candidates and these are then ordered according to their total scores. It is assumed that $s_{m} \geqslant s_{m-1} \geqslant \ldots \geqslant s_{1}$ and that $s_{m}>s_{1}$. At a preference $p(i)$ voter $i$ would hand out score $s_{m}$ to his best candidate, $s_{m-1}$ to his second best and so on. So, his worst candidate receives score $s_{1}$. Let $\operatorname{rank}(x, p(i))=|\{y \in A:(x, y) \in p(i)\}|$ be the rank of candidate $x$ at preference $p(i)$. Then of course the rank of the best candidate is equal to $m$ the number of candidates and that of the worst candidate is equal to one. Voter $i$ assigns the scores as follows $s(x, p(i))=s_{\operatorname{rank}(x, p(i))}$ and total score for candidate $x$ at profile $p$ is now $s(x, p)=\sum_{i \in N} s(x, p(i))$. Define score rule $F_{s}$, with scores $s$, for candidates $x$ and $y$ and an arbitrary profile $p$ as follows

$$
(x, y) \in F_{s}(p) \text { if and only if } s(x, p) \geqslant s(y, p)
$$

In order to show that $F_{s}(\widehat{p})=\widehat{R}$, let $(x, y) \in \widehat{R}$ with $x \neq y$. It is sufficient to show that $s(x, \widehat{p})>s(y, \widehat{p})$. For numbers $v$ and $w$ let $\mathbb{L}_{x y}^{v w}=\left\{R \in \mathbb{L}_{x y}: \operatorname{rank}(x, R)=v\right.$ and $\left.\operatorname{rank}(y, R)=w\right\}$. Note that

$$
\begin{aligned}
s(x, \widehat{p}) & =\sum_{v=2}^{v=m} \sum_{w=1}^{w=v-1}\left(\sum_{R \in \mathbb{L}_{x y}^{v w}} s_{v} \cdot f(R, \widehat{p})+\sum_{R \in \mathbb{L}_{y x}^{v w}} s_{w} \cdot f(R, \widehat{p})\right) \\
& =\sum_{v=2}^{v=m} \sum_{w=1}^{w=v-1}\left(\sum_{R \in \mathbb{L}_{x y}^{v w}} s_{v} \cdot f(R, \widehat{p})+s_{w} \cdot f\left(\tau_{x y} R, \widehat{p}\right)\right)
\end{aligned}
$$

and similarly

$$
s(y, \widehat{p})=\sum_{v=2}^{v=m} \sum_{w=1}^{w=v-1}\left(\sum_{R \in \mathbb{L}_{x y}^{v w}} s_{v} \cdot f\left(\tau_{x y} R, \widehat{p}\right)+s_{w} \cdot f(R, \widehat{p})\right) .
$$

Therefore it is sufficient to show that for all numbers $v$ and $w$, with $v>w$, and all $R \in \mathbb{L}_{x y}^{v w}$

$$
s_{v} \cdot f(R, \widehat{p})+s_{w} \cdot f\left(\tau_{x y} R, \widehat{p}\right) \geqslant s_{v} \cdot f\left(\tau_{x y} R, \widehat{p}\right)+s_{w} \cdot f(R, \widehat{p}),
$$

and that at least one of these inequalities is strict. The latter equality is equivalent to

$$
\left(s_{v}-s_{w}\right) \cdot\left(f(R, \widehat{p})-f\left(\tau_{x y} R, \widehat{p}\right)\right) \geqslant 0
$$

Because $v>w$ we have that $s_{v} \geqslant s_{w}$, where by definition $s_{m}>s_{1}$. Lemma ?? yields that $f(R, \hat{p})-$ $f\left(\tau_{x y} R, \widehat{p}\right)>0$. So, the weak inequality follows readily. Strictness follows because at some preference $R$ candidate $x$ is ordered best and candidate $y$ is ordered worst. 


\section{Sufficient Conditions for Choosing the Mode}

Here we state a set of sufficient conditions for arbitrary rules such that these have the mode as the outcome at unimodal preference distributions. As many rules satisfy these conditions we consider them as weak. Monotonicity as well as (positive) discrimination is closely related to the result spelled out by Lemma ??.

Theorem 1 Let $p$ be a unimodal profile with mode $\widehat{R}$. Then $F(p)=\widehat{R}$ for a rule $F$ from $\mathbb{L}^{N}$ to $\mathbb{W}$ in each of the following two cases:

1. F is positively discriminating;

2. F is anonymous, neutral, monotone and discriminating.

Proof It is sufficient to prove that $(x, y) \in F(p)$ and $(y, x) \notin F(p)$. Let $R \in \mathbb{L}_{x y}$. As Lemma ?? implies that $d\left(\widehat{R}, \tau_{x y} R\right)>d(\widehat{R}, R)$, because $p$ is unimodal it follows for all $R \in \mathbb{L}_{x y}$, that $f(R, p)>f\left(\tau_{x y} R, p\right)$. But then $(x, y) \in F(p)$ and $(y, x) \notin F(p)$ because $F$ is positively discriminating.

Lemma ?? together with part (1) imply part (2).

Lemma 3 Let $F$ be a rule from $\mathbb{L}^{N}$ to $\mathbb{W}$ which is neutral, anonymous, monotone and discriminating. Then $F$ is positively discriminating.

Proof In order to prove the second case let $F$ be anonymous, neutral, monotone and discriminating. Let $p$ be a profile and $x$ and $y$ be different candidates such that for all $R \in \mathbb{L}_{x y}$ frequencies $f(R, p)$ are strictly greater than $f\left(\tau_{x y} R, p\right)$. It is sufficient to prove that $(x, y) \in F(p)$ and $(y, x) \notin F(p)$. By discrimination of $F$ we have that $(x, y) \notin F(p)$ or $(y, x) \notin F(p)$. To the contrary suppose $(x, y) \notin F(p)$. It is sufficient to prove a contradiction. As $F(p)$ is a weak order, hence complete, $(y, x) \in F(p)$ and $(x, y) \notin F(p)$. Furthermore, neutrality implies $(y, x) \notin F\left(\tau_{x y} p\right)$ and $(x, y) \in F\left(\tau_{x y} p\right)$. Now for all $R \in \mathbb{L}_{x y}$

$$
f\left(\tau_{x y} R, \tau_{x y} p\right)=f(R, p)>f\left(\tau_{x y} R, p\right)=f\left(R, \tau_{x y} p\right) .
$$

This means that at profile $\tau_{x y} p$ every $R \in \mathbb{L}_{x y}$ is outnumbered by the frequency of $\tau_{x y} R$ which is in $\mathbb{L}_{y x}$. Therefore at $\tau_{x y} p$ we can distinguish a non-empty subset $S$ of $N$ such that

1. $f\left(R,\left.\left(\tau_{x y} p\right)\right|_{N-S}\right)=f\left(\tau_{x y} R,\left.\left(\tau_{x y} p\right)\right|_{N-S}\right)$ for all $R \in \mathbb{L}_{x y}$ and

2. $(x, y) \notin \tau_{x y} p(i)$ for all $i \in S$.

Consider profile $q$ such that $q(j)=\tau_{x y} p(j)$ for all $j \in N-S$ and $q(i)=\tau_{x y}\left(\tau_{x y} p(i)\right)=p(i)$ for all $i \in S$. Monotonicity and $(y, x) \notin F\left(\tau_{x y} p\right)$ imply $(y, x) \notin F(q)$. Hence, $(x, y) \in F(q)$ and $(y, x) \notin F(q)$. Next we show that $f(R, q)=f(R, p)$ for all $R$ in $\mathbb{L}$. For $R$ in $\mathbb{L}_{x y}$ this follows because $f(R, q)=f\left(R,\left.q\right|_{S}\right)+$ $f\left(R,\left.q\right|_{N-S}\right)=f\left(R,\left.p\right|_{S}\right)+f\left(R,\left.\left(\tau_{x y} p\right)\right|_{N-S}\right)=f\left(R,\left.p\right|_{S}\right)+f\left(\tau_{x y} R,\left.\left(\tau_{x y} p\right)\right|_{N-S}\right)=f\left(R,\left.p\right|_{S}\right)+f\left(R,\left.p\right|_{N-S}\right)=$ $f(R, p)$. For $R$ in $\mathbb{L}_{y x}$ note that $f\left(R,\left.p\right|_{S}\right)=f\left(R,\left.q\right|_{S}\right)=0$ as $(y, x) \notin p(i)$ by the choice of $S$. So, the sequence of equations above alters to $f(R, q)=f\left(R,\left.q\right|_{S}\right)+f\left(R,\left.q\right|_{N-S}\right)=f\left(R,\left.\left(\tau_{x y} p\right)\right|_{N-S}\right)=f\left(\tau_{x y} R,\left.\left(\tau_{x y} p\right)\right|_{N-S}\right)=$ $f\left(R,\left.p\right|_{S}\right)+f\left(R,\left.p\right|_{N-S}\right)=f(R, p)$. But then by anonymity we have $F(q)=F(p)$, which cannot be because $(x, y) \in F(q)$ and $(x, y) \notin F(p)$.

The following example shows that the conditions in Theorem ?? case 2 are logically independent.

Example 4 Independence of anonymity, neutrality, monotonicity and discrimination

Let the dictatorial rule $F_{\text {dict }, i}$ with dictator $i$ be defined for a profile $p$ as $F_{\text {dict }, i}(p)=p(i)$. So, this rule assigns the preference of voter $i$ independent of the preferences of all other voters. Note that $F_{d i c t, i}$ is neutral, monotone and discriminating but of course not anonymous.

For a weak order $R$ on $A$ let the constant rule $F_{\text {const }, R}$ be defined for a profile $p$ as $F_{\text {const }, R}(p)=R$. So, this rule assigns relation $R$ independent of the preferences of the voters. Note that if $R \in \mathbb{L}$, then 
$F_{\text {const }, R}$ is anonymous, monotone and discriminating but not neutral, where if $R=A \times A$, then $F_{\text {const }, A \times A}$ is anonymous, neutral and monotone, but not discriminating.

Let the reverse transitive closure of pairwise majority rule $F_{\text {odd }}$ be defined for a profile $p$ and a pair of candidates $x$ and $y$ as follows, $(x, y) \in F_{o d d}(p)$ if and only if there are $y=z_{0}, z_{1}, \ldots, z_{k}=x$ such that for all $0<j \leqslant k$

$$
\left|\left\{i \in N:\left(z_{j}, z_{j-1}\right) \in p(i)\right\}\right| \geqslant\left|\left\{i \in N:\left(z_{j-1}, z_{j}\right) \in p(i)\right\}\right| .
$$

It is straightforward to prove that $F_{o d d}$ is neutral, anonymous and discriminating. It is clearly not monotone.

It is clear that for instance Condorcet consistent or pairwise rules as well as score rules are positively discriminating and therefore by Theorem ?? their outcome equals the mode at unimodal distributed profiles.

An application of the second case of Theorem ?? can be found in the following example.

\section{Example 5 Score-wise Elimination}

Consider score rules introduced in Example ??. Let $s^{k}=\left(s_{1}^{k}, s_{2}^{k}, \ldots, s_{k}^{k}\right) \in \mathbb{R}^{k}$ for each $k$ from 1 to $m$, with $s_{k}^{k} \geqslant s_{k-1}^{k} \geqslant \ldots \geqslant s_{1}^{k}$ and $s_{k}^{k}>s_{1}^{k}$. Based on these score vectors the rule sequentially eliminates those alternatives which have lowest score. Here for a subset $B$ of $A$ the scores are determined for an arbitrary candidate $b$ in $B$ and profile $p$ as follows

$$
\operatorname{score}\left(s^{k}, b,\left.p\right|_{B}\right)=\sum_{i \in N} \operatorname{score}\left(s^{k}, b,\left.p\right|_{B}\right),
$$

where $|B|=k, \operatorname{score}\left(s^{k}, b,\left.p\right|_{B}\right)=s_{r\left(x,\left.p(i)\right|_{B}\right)}^{k}$ and $r\left(x,\left.p(i)\right|_{B}\right)=\left|\left\{x \in B:\left.(b, x) \in p(i)\right|_{B}\right\}\right|$. So, in round 0 set $A_{0}=A$ and

$$
C_{0}(p)=\left\{a \in A: \operatorname{score}\left(s^{m}, x, p\right) \geqslant \operatorname{score}\left(s^{m}, a, p\right) \text { for all } x \in A\right\} .
$$

Now for each proceeding round $l>0$ define $A_{l}=A_{l-1}-C_{l-1}(p)$, and take

$$
\begin{aligned}
& C_{l}(p)=\left\{a \in A_{l}: \operatorname{score}\left(s^{k}, x,\left.p\right|_{A_{l}}\right) \geqslant \operatorname{score}\left(s^{k}, a,\left.p\right|_{A_{l}}\right) \text { for all } x \in A_{l}\right\} \\
& \quad \text { and } A_{l+1}=A_{l}-C_{l}(p) \text { if }\left|A_{l}\right|=k>0 \\
& C_{l}(p)=A_{l+1}=\emptyset \text { else. }
\end{aligned}
$$

The outcome of the score-wise elimination rule $F_{\text {elimination }}$ is now defined for an arbitrary pair of candidates and profile $p$ as follows

$$
(x, y) \in F_{\text {elimination }}(p) \text { if and only if } y \in A_{l} \text { implies } x \in A_{l} \text { for all } l \geq 0 .
$$

In case $s^{k}$ equals the anti-plurality scores, i.e. $(1,1,1, \ldots, 1,1,0)$ for all $k$, this score-wise elimination equals Coombs rule, in case it equals Borda score, i.e. $(k, k-1, \ldots, 2,1)$ it equals Nanson rule. The class is much richer though as for instance resemblance between the score vectors at different rounds of $k$ need not exist. We end this example by arguing that all score-wise elimination rules assign the mode to unimodal profiles. The argument is based on Theorem ?? case 2. By definition these elimination rules are neutral and anonymous. Note that these rules are monotone. Taking profiles $p$ and $q$ and candidates $x$ and $y$ as in the definition. At every subset of candidates in which these two are present the scores of all others is not affected and that of $x$ is weakly increased where that of $y$ is weakly decreased while going from $p$ to $q$. Next we show that these rules are discriminating. Let at profile $p$ and at subset $B$, with 
$|B|=k$, candidates $x$ and $y$ both be eliminated at round $t$, where $p, x$ and $y$ are chosen as in the definition of discrimination.

Then scores of these at $\left.p\right|_{B}$ are equal. Now as $s_{k}^{t}>s_{1}^{t}$ it cannot be that $f(R, p)>f\left(\tau_{x y} R, p\right)$ for all linear orders $R \in \mathbb{L}_{x y}$. As this would yield $f\left(\left.R\right|_{B},\left.p\right|_{B}\right)>f\left(\left.\left(\tau_{x y} R\right)\right|_{B}, p_{\left.\right|_{B}}\right)$ for all linear orders $R \in \mathbb{L}_{x y}$, which can both not hold because of score being equal at $\left.p\right|_{B}$ where $s_{t}^{t}>s_{1}^{t}$. Similarly not $f(R, p)>f\left(\tau_{x y} R, p\right)$ for all linear orders $R \in \mathbb{L}_{y x}$. So, we have that these rules are discriminating. By Theorem ?? case 2 they assign the mode at unimodal distributions.

\section{Robustness of the Mode-selection Property}

So far we have studied behaviour of well-known rules under the assumption that preference profiles have a unimodal distribution. In this section we relax this assumption and try to analyse how sensitive the results of Examples ?? and ?? are to perturbations of unimodal distributions. We confine our study here to specific cases of perturbations and specific rules. Of course we tried to deduce the similar results for more abstract rules like a subclass of score rules or abstract rules satisfying the sufficient conditions of Theorem ?? Case 2, but were not able to do so. As for rules we only consider Borda, plurality and Condorcet consistent rules. So, within the class of score rules we take two 'extreme' cases: all scores are different with equal margins (Borda) and except for the highest score all other scores are equal (Plurality). We further only consider perturbations of the following kind. There is a linear order, say $R^{*}$, and a real number $v$ such that

1. Frequencies are constant at any given distance from $R^{*}$. So, $f\left(R^{1}, p\right)=f\left(R^{2}, p\right)$ whenever $d\left(R^{1}, R^{*}\right)=$ $d\left(R^{2}, R^{*}\right)$ for all linear orders $R^{1}$ and $R^{2}$.

2. For linear orders $R^{1}, R^{2}$ and $R^{3}$, with $d\left(R^{1}, R^{*}\right)<d\left(R^{2}, R^{*}\right) \leqslant v<d\left(R^{3}, R^{*}\right)$ we have $f\left(R^{1}, p\right)>$ $f\left(R^{2}, p\right)>f\left(R^{3}, p\right)$.

Such distribution behave like a unimodal distribution with mode $R^{*}$ till distance $v$ from the mode and thereafter frequency is bounded by the frequency at distance $\lfloor v\rfloor^{\square}$ but does not have to decline like in unimodal distributions. We call such distributions $v$-tail perturbed unimodal distributions around $R^{*}$.

Let $\rho$ denote the radius distance, i.e. $\rho=\frac{1}{2} \delta$, where $\delta=\left(\begin{array}{c}m \\ 2\end{array}\right)$ denotes the diameter distance of $\mathbb{L}$. In all results we will take $\rho \leqslant v$. So, seen from $R^{*}$ these perturbations only occur in the second half of the set of linear orders. At such distributions frequency depends on the distance to $R^{*}$. Further, at distances larger than radius distance, say $\delta-k$, frequency is lower than at the 'opposite' distance $k$.

The analysis needs some combinatorial results which are discussed in the Appendix. Also some additional notations related to a (distance) $k$, candidates $x$ and $y$, linear orders $R$ and rank number $r$ are needed.

- $\mathbb{L}^{k}=\left\{R \in \mathbb{L}: d\left(R, R^{*}\right)=k\right\}$, i.e. the set of all linear orders on distance $k$ from $R^{*}$. This set is equal to $\left\{R \in \mathbb{L}: d\left(R,-R^{*}\right)=\delta-k\right\}$. Further, $l(k)=\left|\mathbb{L}^{k}\right|$ denotes the cardinality of $\mathbb{L}^{k}$.

- $\mathbb{L}_{x y}^{k}=\mathbb{L}_{x y} \cap \mathbb{L}^{k}$, i.e. the set of linear orders preferring $x$ to $y$ on distance $k$ from $R^{*}$ and $l(k, x y)=\left|\mathbb{L}_{x y}^{k}\right|$ denotes the cardinality of $\mathbb{L}_{x y}^{k}$.

- Also throughout this section we write $f(R)$ instead of $f(R, p)$ and also write $f(k)$ to indicate the frequency of linear orders on distance $k$ from $R^{*}$.

- $l(k, x, r)$, i.e. number of all linear orders on distance $k$ from $R^{*}$, where rank of $x$ is $r$.

- Let $\bar{l}, \bar{\delta}$ and $\bar{\rho}$ refer function $l$ in the case of $m-1$ candidates, the diameter and the radius in that case respectively.

\footnotetext{
${ }^{7}\lfloor v\rfloor$ denotes the largest integer less than or equal to $v$.
} 
Example 6 Example of a tail-perturbed unimodal distribution with 4 candidates $a, b, c, d$.

The following figure shows an example of a tail-perturbed unimodal distribution with 4 candidates. Suppose $a b c d$ is the mode of the distribution. Thereafter frequencies decline till distance 3 (i.e., $v=3$ ). After that frequencies fluctuate having an upper bound : the frequency at distance 3.

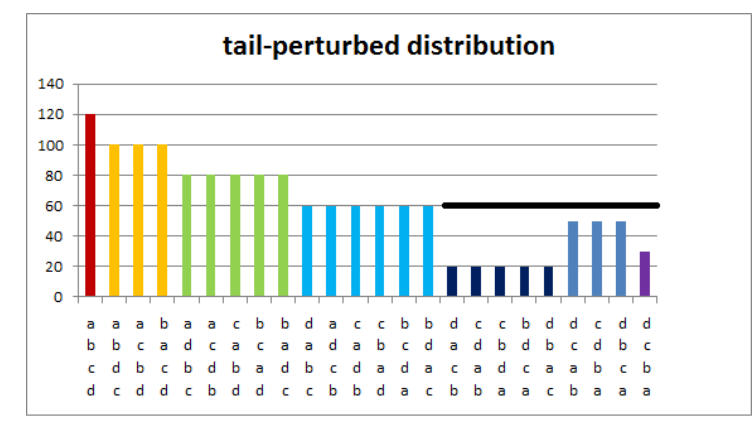

Fig. 2: Tail-perturbed unimodal distribution

In the following theorem we show that at such perturbations Condorcet consistent rules choose $R^{*}$.

Theorem 2 Let $p$ have a $\rho$-tail perturbed unimodal distributions around $R^{*}$. Then $R^{*}$ is the Condorcet order at $p$.

Proof Let $(x, y) \in R^{*}$ for different candidates $x$ and $y$. It is sufficient to prove that $\sum_{R \in \mathbb{L}_{x y}} f(R)-f\left(\tau_{x y} R\right)>$ 0 .

Note that

$$
\sum_{R \in \mathbb{L}_{x y}} f(R)-f\left(\tau_{x y} R\right)=\sum_{k=0}^{\delta}\left(\sum_{R \in \mathbb{L}_{x y}^{k}} f(R)-\sum_{R \in \mathbb{L}_{y x}^{k}} f(R)\right)
$$

As for linear order $R$ on distance $\rho$ from $R^{*}$ linear order $-R$ is also on distance $\rho$ from $R^{*}$ and frequencies are constant over a given distance we may exclude $k=\rho$ from this sum and split it up to yield

$$
\begin{aligned}
\sum_{R \in \mathbb{L}_{x y}} f(R)-f\left(\tau_{x y} R\right)= & \sum_{0 \leq k<\rho}\left(\sum_{R \in \mathbb{L}_{x y}^{k}} f(R)-\sum_{R \in \mathbb{L}_{y x}^{k}} f(R)\right) \\
& +\sum_{0 \leq k<\rho}\left(\sum_{R \in \mathbb{L}_{x y}^{\delta-k}} f(R)-\sum_{R \in \mathbb{L}_{y x}^{\delta-k}} f(R)\right) \\
= & \sum_{0 \leq k<\rho} l(k, x y) f(k)-l(k, y x) f(k) \\
& +\sum_{0 \leq k<\rho} l(\delta-k, x y) f(\delta-k)-l(\delta-k, y x) f(\delta-k) .
\end{aligned}
$$

Where the latter equality follows by the assumptions on the frequency distribution. As further $l(k, x y)=$ $l(\delta-k, y x)$ because $R \in \mathbb{L}_{x y}^{k}$ if and only if $-R \in \mathbb{L}_{y x}^{\delta-k}$ we have 


$$
\begin{aligned}
\sum_{R \in \mathbb{L}_{x y}} f(R)-f\left(\tau_{x y} R\right) & =\sum_{0 \leq k<\rho} l(k, x y)[f(k)-f(\delta-k)]+l(k, y x)[(f(\delta-k)-f(k)] \\
& =\sum_{0 \leq k<\rho}[l(k, x y)-l(k, y x)][f(k)-f(\delta-k)] .
\end{aligned}
$$

Now by Lemma ?? $l(k, x y) \geqslant l(k, y x)$ for all $0 \leq k \leq \rho$. The assumptions on the distribution show that $f(k)-f(\delta-k)$ is positive for all $0 \leq k \leq \rho$. For $k=0$ it is obvious that $l(k, x y)>l(k, y x)$ and $f(k)>f(\delta-k)$. So, $\sum_{R \in \mathbb{L}_{x y}} f(R)-f\left(\tau_{x y} R\right)$ is strictly positive.

Remark 1 In Theorem ?? the assumption on frequencies being constant on a given distance from $R^{*}$ can be relaxed and further looking at the proof frequencies at distance $\rho$ from $R^{*}$ need not be an upper bound for frequencies at greater distances. That is in the same way we can prove that $R^{*}$ is the Condorcet order if frequencies satisfy

$$
\min _{R \in \mathbb{L}_{x y}^{k}} f(R)+\min _{R \in \mathbb{L}_{x y}^{\delta-k}} f(R)>\max _{R \in \mathbb{L}_{y x}^{k}} f(R)+\max _{R \in \mathbb{L}_{y x}^{\delta-k}} f(R) \text { for all } 0 \leq k<\rho .
$$

As by the second equation in the foregoing proof we have

$$
\begin{aligned}
& \sum_{R \in \mathbb{L}_{x y}} f(R)-f\left(\tau_{x y} R\right) \geqslant \sum_{0 \leq k<\rho} l(k, x y) \cdot \min _{R \in \mathbb{L}_{x y}^{k}} f(R)-l(k, y x) \cdot \max _{R \in \mathbb{L}_{y x}^{k}} f(R) \\
& l(-k, x y) \cdot \min _{R \in \mathbb{L}_{x y}^{-k}} f(R)-l(-k, y x) \cdot \max _{R \in \mathbb{L}_{y x}^{-k}} f(R) \\
&=\sum_{0 \leq k<\rho} l(k, x y) \cdot\left(\min _{R \in \mathbb{L}_{x y}^{k}} f(R)-\max _{R \in \mathbb{L}_{y x}^{-k}} f(R)\right) \\
&-l(k, y x) \cdot\left(\max _{R \in \mathbb{L}_{y x}^{k}} f(R)-\min _{R \in \mathbb{L}_{x y}^{-k}} f(R)\right) .
\end{aligned}
$$

Now as $l(k, x y) \geqslant l(k, y x)$ and the assumption the result follows.

Example 7 Performance of Condorcet consistent rules under tail perturbed unimodal distribution

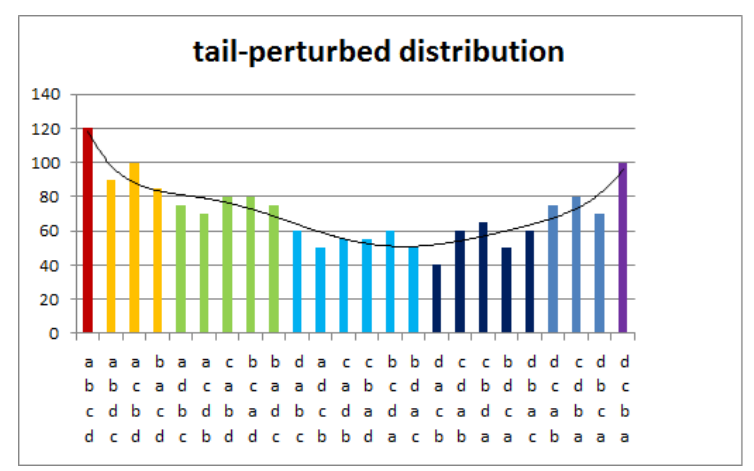

Fig. 3: Robustness of Condorcet consistent rules 
In the above figure ??, we show a voting situation with tail-perturbed unimodal frequencies satisfying the conditions given in remark ??. Here we consider a voting situation with 4 candidates $a, b, c, d$. abcd is the mode of the distribution. Thereafter frequencies decline till radius distance $\rho=3$. Here it is also possible to have different frequencies at the same distance from the mode. After radius frequencies are just bounded by the minimum frequency at the same distance from $d c b a$, the reverse mode. Also, till the radius, maximum frequency at any distance from the mode is lesser than the minimum frequency at any distances closer to the mode.

Next we check for sensitivity of the Borda rule. Borda rule is based on Borda score. Borda score of a candidate $x$ is the number of candidates below $x$ in a preference $R$, summed over all preferences. We show that for perturbed distributions discussed above, the Borda rule chooses $R^{*}$ as the outcome.

Theorem 3 Let $p$ have a $\rho$-tail perturbed unimodal distributions around $R^{*}$. Then Borda rule assigns outcome $R^{*}$ to $p$.

Proof Let scores $s_{t}$ be the Borda scores, i.e. $s_{t}=t$ for all $1 \leqslant t \leqslant m$. Take $(x, y) \in R^{*}$ arbitrary where $x$ and $y$ are different candidates. It is sufficient to prove that $s(x, p)>s(y, p)$. Note for each distance $k$ to $R^{*}$ Borda score of candidate $x$ satisfies

$$
\sum_{z \in A} l(k, x z)=\sum_{r=1}^{m} r \cdot l(k, x, r)
$$

So, Borda score of candidate $x$ equals

$$
\begin{aligned}
s(x, p) & =\sum_{k=0}^{\delta} \sum_{z \in A} f(k) \cdot l(k, x z) \\
& =\sum_{0 \leq k<\rho} \sum_{z \in A} f(k) \cdot l(k, x z)+f(\delta-k) \cdot l(\delta-k, x z) .
\end{aligned}
$$

Where the latter equation follows because $R$ is on distance $\rho$ from $R^{*}$ then so is $-R$. Noting that $l(\delta-k, b a)=l(k, a b)$ for all candidates $a$ and $b$ and all distances $k$ between 0 and $\rho$ we have

$$
\begin{aligned}
s(x, p) & =\sum_{0 \leq k<\rho} \sum_{z \in A} f(k) \cdot l(k, x z)+f(\delta-k) \cdot l(\delta-k, x z) \\
& =\sum_{0 \leq k<\rho} \sum_{z \in A} f(k) \cdot l(k, x z)+f(\delta-k) \cdot l(\delta-k)-f(\delta-k) \cdot l(\delta-k, z x) \\
& =\sum_{0 \leq k<\rho} \sum_{z \in A}[f(k)-f(\delta-k)] \cdot l(k, x z)+f(\delta-k) \cdot l(\delta-k) .
\end{aligned}
$$

Hence,

$$
s(x, p)-s(y, p)=\sum_{0 \leq k<\rho} \sum_{z \in A}[f(k)-f(\delta-k)] \cdot[l(k, x z)-l(k, y z)] .
$$

Note that for $k=0$ we obviously have that $\sum_{z \in A} l(k, x z)-l(k, y z)>0$. So, by the assumptions on frequency distribution $f$ and Lemma ?? we are done.

Similar to the case with Condorcet consistent rules, here also we mention a more general version of the result in the following Remark. 
Remark 2 Looking at the proof of Theorem ?? it is clear that the same result can be deduced in case the distribution satisfies

$$
f(k)>f(\delta-k) \text { for all } 0 \leq k<\rho .
$$

Example 8 Performance of Borda rule under tail perturbed unimodal distribution

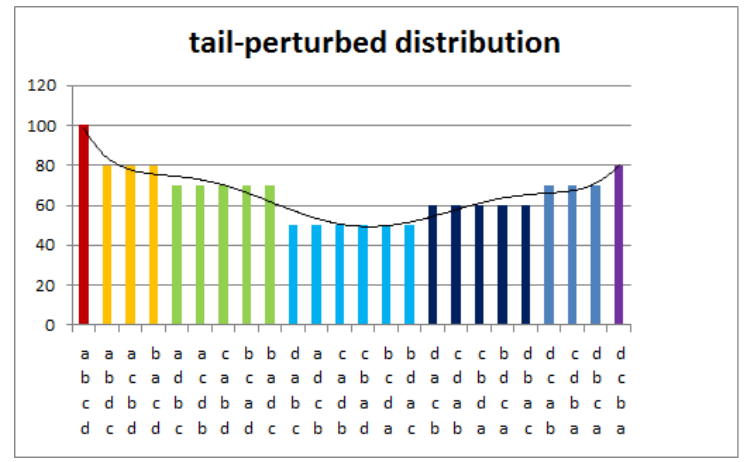

Fig. 4: Robustness of Borda rule

In this example we show a voting situation with tail-perturbed unimodal frequencies satisfying the condition given in remark ??. Unlike example ??, here we have equal frequencies at the same distance from the mode.

Finally we show that the outcome of plurality rule at $v$-tail perturbed unimodal distributions towards $R^{*}$ is equal to $R^{*}$. We need $v$ to be equal to $\bar{\mu}=\frac{1}{2}\left(\begin{array}{c}m-1 \\ 2\end{array}\right)+m-\frac{3}{2}$.

Plurality rule is based on plurality score. That is the number of times a candidate is at the top of voter's preferences, i.e. having rank $m$ at the preference of an agent. It ranks the candidates according to their plurality scores. Consider profile $p$ having $\bar{\mu}$-tail perturbed unimodal distributions towards $R^{*}$. Let $p l(x)$ denote this plurality score for candidate $x$. In this situation we have

$$
p l(x)=\sum_{k=0}^{\delta} l(k, x, m) \cdot f(k) .
$$

Let $r_{x}$ be the rank of $x$ in $R^{*}$. Then for any distance $k \geqslant u_{x}$, with $u_{x}=m-r_{x}$ we have that $l(k, x, m)=$ $\bar{l}\left(k-u_{x}\right)$. Where $\bar{l}\left(k-u_{x}\right)$ denotes the number of preferences at distance $k-u_{x}$ from $\bar{R}^{*}$ the restriction of $R^{*}$ to $A-\{x\}$. This is because it needs precisely $u_{x}$ swaps to bring $x$ to the top at a preference when at start its rank is $r_{x}$. Denoting the diameter of the set of linear orders on $m-1$ candidates by $\bar{\delta}$ we therefore have

$$
p l(x)=\sum_{k=u_{x}}^{\bar{\delta}+u_{x}} \bar{l}\left(k-u_{x}\right) \cdot f(k) .
$$

The above sum is over all $k \in\left\{u_{x}, u_{x}+1, \ldots, \bar{\delta}+u_{x}\right\}$, because if $k$ is outside this range, $\bar{l}\left(k-u_{x}\right)$ is not defined. Now we can prove the following result on plurality rule.

Theorem 4 Let $p$ have a $\bar{\mu}$-tail perturbed unimodal distributions around $R^{*}$. Then the outcome of Plurality rule at p equals $R^{*}$. 
Proof Let $x$ and $y$ be different candidates such that $(x, y) \in R^{*}$. It is sufficient to prove $p l(x)-p l(y)>0$. Let $r_{x}$ and $r_{y}$ denote the rank of $x$ and $y$ in $R^{*}$ respectively. Let $u_{x}=m-r_{x}$ and $u_{y}=m-r_{y} . r_{x}>r_{y}$ implies $u_{x}<u_{y}$. Then $p l(x)-p l(y)$ is equal to

$$
\sum_{k=u_{x}}^{\bar{\delta}+u_{x}} \bar{l}\left(k-u_{x}\right) \cdot f(k)-\sum_{k=u_{y}}^{\bar{\delta}+u_{y}} \bar{l}\left(k-u_{y}\right) \cdot f(k) .
$$

Take $\mu_{x y}$ the average of $\bar{\rho}+u_{x}$ and $\bar{\rho}+u_{y}$, so $\mu_{x y}=\bar{\rho}+\frac{1}{2}\left(u_{x}+u_{y}\right)$, where $\bar{\rho}$ is the radius distance of the set of linear orders on $m-1$ candidates. Defining $\bar{l}(k)$ to be zero if $k$ is not in the interval $[0, \bar{\delta}]$ we have that $p l(x)-p l(y)$ is equal to

$$
\sum_{u_{x} \leqslant k \leq \mu_{x y}}\left[\bar{l}\left(k-u_{x}\right)-\bar{l}\left(k-u_{y}\right)\right] \cdot f(k)+\sum_{\mu_{x y}<k \leq \bar{\delta}+u_{y}}\left[\bar{l}\left(k-u_{x}\right)-\bar{l}\left(k-u_{y}\right)\right] \cdot f(k) .
$$

For $k=\mu_{x y}=\bar{\rho}+\frac{1}{2}\left(u_{x}+u_{y}\right)$ we have $\bar{l}\left(k-u_{x}\right)=\bar{l}\left(\bar{\rho}+\frac{1}{2}\left(u_{y}-u_{x}\right)\right)$ and $\bar{l}\left(k-u_{y}\right)=\bar{l}\left(\bar{\rho}-\frac{1}{2}\left(u_{y}-u_{x}\right)\right)$. As $\bar{l}$ is symmetric around $\bar{\rho}$ it follows that $\left[\bar{l}\left(k-u_{x}\right)-\bar{l}\left(k-u_{y}\right)\right] \cdot f(k)$ is zero for $k=\mu_{x y}$. So, pl( $\left.x\right)-$ $p l(y)$ is equal to

$$
\sum_{u_{x} \leqslant k<\mu_{x y}}\left[\bar{l}\left(k-u_{x}\right)-\bar{l}\left(k-u_{y}\right)\right] \cdot f(k)+\sum_{\mu_{x y}<k \leq \bar{\delta}+u_{y}}\left[\bar{l}\left(k-u_{x}\right)-\bar{l}\left(k-u_{y}\right)\right] \cdot f(k)
$$

Substituting $k^{\prime}=\bar{\delta}+u_{x}+u_{y}-k$,

$$
\begin{aligned}
= & \sum_{u_{x} \leqslant k<\mu_{x y}}\left[\bar{l}\left(k-u_{x}\right)-\bar{l}\left(k-u_{y}\right)\right] \cdot f(k)+ \\
& \sum_{u_{x} \leqslant k^{\prime}<\mu_{x y}}\left[\bar{l}\left(\left(\bar{\delta}+u_{y}\right)-\left(k^{\prime}-u_{x}\right)-u_{x}\right)-\bar{l}\left(\left(\bar{\delta}+u_{y}\right)-\left(k^{\prime}-u_{x}\right)-u_{y}\right)\right] \cdot f\left(\left(\bar{\delta}+u_{y}\right)-\left(k^{\prime}-u_{x}\right)\right) .
\end{aligned}
$$

As

$$
\begin{aligned}
& \bar{l}\left(\left(\bar{\delta}+u_{y}\right)-\left(k^{\prime}-u_{x}\right)-u_{x}\right)-\bar{l}\left(\left(\bar{\delta}+u_{y}\right)-\left(k^{\prime}-u_{x}\right)-u_{y}\right) \cdot f\left(\left(\bar{\delta}+u_{y}\right)-\left(k^{\prime}-u_{x}\right)\right) \\
& =\left[\bar{l}\left(\bar{\delta}+u_{y}-k^{\prime}\right)-\bar{l}\left(\bar{\delta}+u_{x}-k^{\prime}\right)\right] \cdot f\left(\bar{\delta}+u_{x}+u_{y}-k^{\prime}\right)
\end{aligned}
$$

and $\bar{l}\left(\bar{\delta}+u_{y}-k^{\prime}\right)=\bar{l}\left(k^{\prime}-u_{y}\right)$ and $\bar{l}\left(\bar{\delta}+u_{x}-k^{\prime}\right)=\bar{l}\left(k^{\prime}-u_{x}\right)$ it follows that

$$
\begin{aligned}
p l(x)-p l(y)= & \sum_{u_{x} \leqslant k<\mu_{x y}}\left[\bar{l}\left(k-u_{x}\right)-\bar{l}\left(k-u_{y}\right)\right] \cdot f(k)+ \\
& \sum_{u_{x} \leqslant k<\mu_{x y}}\left[\bar{l}\left(k-u_{y}\right)-\bar{l}\left(k-u_{x}\right)\right] \cdot f\left(\bar{\delta}+u_{x}+u_{y}-k\right) .
\end{aligned}
$$

So,

$$
p l(x)-p l(y)=\sum_{u_{x} \leqslant k<\mu_{x y}}\left[\bar{l}\left(k-u_{x}\right)-\bar{l}\left(k-u_{y}\right)\right] \cdot\left[f(k)-f\left(\bar{\delta}+u_{x}+u_{y}-k\right)\right] .
$$


By Lemma ?? it follows that the first factor in each term of this latter summation is positive and by the assumptions on $f$ that the second factor is positive. All in all therefore $p l(x)-p l(y)>0$ which completes the proof.

Example 9 Performance of Plurality rule under tail perturbed unimodal distribution

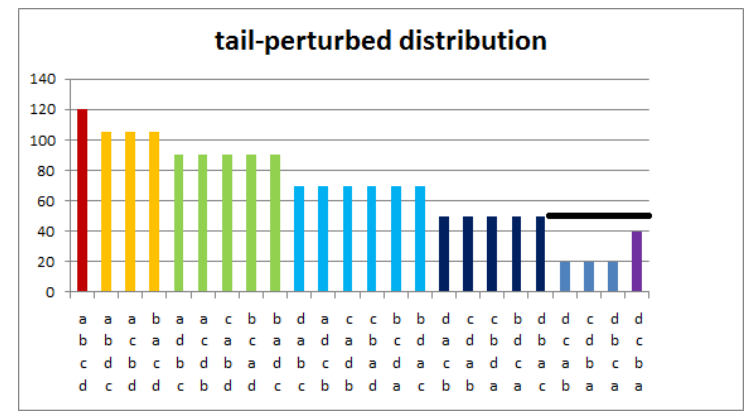

Fig. 5: Robustness of plurality rule

Here is a voting situation with tail-perturbed unimodal frequencies satisfying conditions given in theorem ??. For 4 candidates $a, b, c, d$ we have $\bar{\mu}=4$. So, disturbances happen only after distance 4 from the mode $a b c d$.

It is obvious that these conditions for choosing $R^{*}$ by Condorcet consistent, Borda and plurality rules are not necessary.

\section{Multimodal Frequency Distributions}

Election outcomes often yield vote accumulations around several candidates or parties. It is therefore possible that these consist of several unimodal distributions superposed on each other, where their modes represent these spots of accumulation. In this section we study the case where two unimodal distributions are superposed on each. Here positively discriminating rules choose an outcome between these two modes. The notion of betweenness means that the Kemeny distances between the outcome of the rule to each mode precisely adds up to the distance between these two modi. So, the outcome can be seen as a convex combination (in the Kemeny sense) of the two modes.

Let $N_{1}$ and $N_{2}$ be two disjoint sets of voters. Let $p^{1} \in \mathbb{L}^{N_{1}}$ and $p^{2} \in \mathbb{L}^{N_{2}}$ such that $p^{t}$ is a unimodal profile on $N_{t}$ with mode $R^{t}$ for $t \in\{1,2\}$. Let $N=N_{1} \cup N_{2}$ and $p \in \mathbb{L}^{N}$ defined by $p(i)=p^{t}(i)$ if $i \in N_{t}$ for $t \in\{1,2\}$. In that case $p$ is said to be a superposition of unimodal profiles $p^{1}$ and $p^{2}$. It is straightforward to see that on such superposed profiles different rules may result in different outcomes. On the other hand the following theorem shows that positively discriminating preference rules agree on the intersection of these two modes.

Example 10 Example of multimodal distribution with 3 candidates $a, b, c$. 


\begin{tabular}{|c|c|c|c|}
\hline Preferences & $\mathbf{p}$ & $\mathbf{q}$ & $\mathbf{p + q}$ \\
\hline$a b c$ & 100 & 80 & 180 \\
$a c b$ & 80 & 60 & 140 \\
$b a c$ & 80 & 100 & 180 \\
$c a b$ & 60 & 20 & 80 \\
$b c a$ & 60 & 80 & 140 \\
$c b a$ & 20 & 60 & 80 \\
\hline
\end{tabular}

Table 1: Multimodal distribution
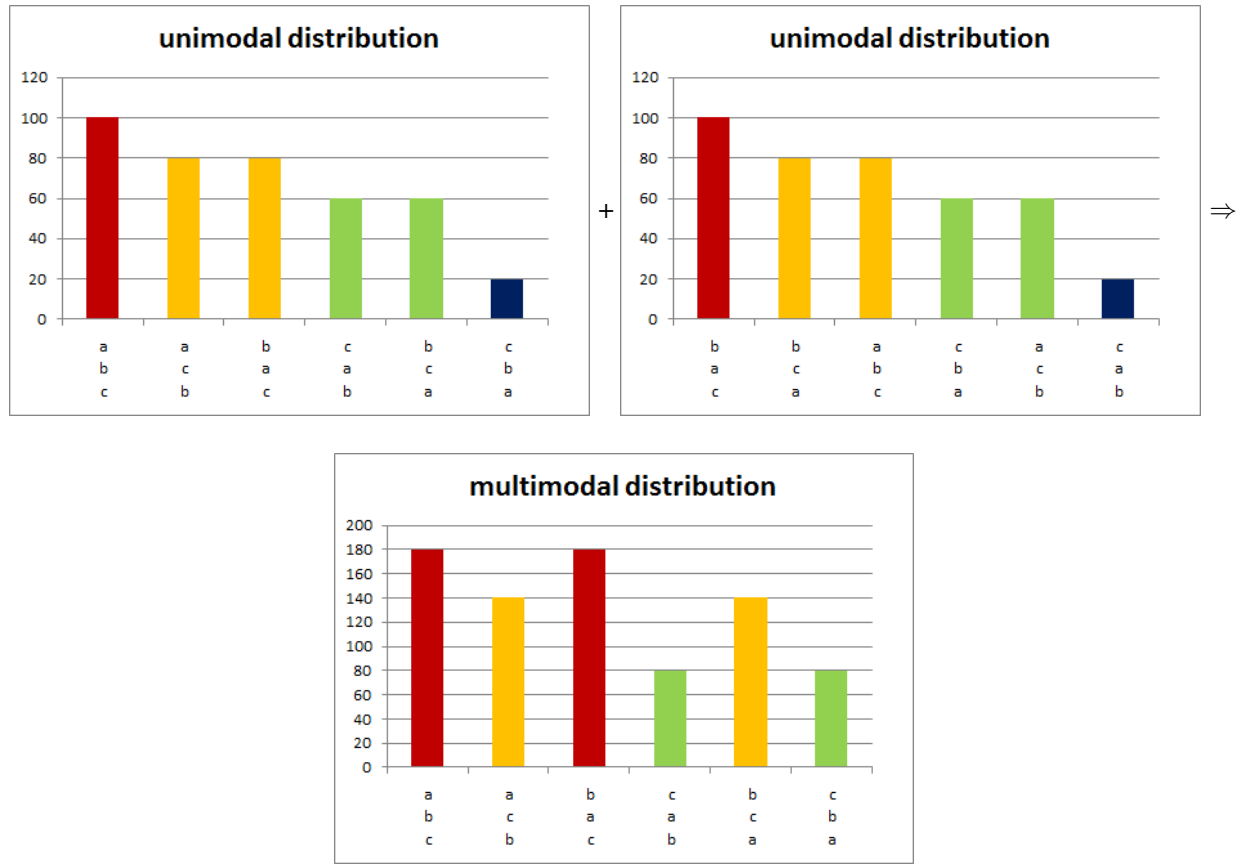

Fig. 6: Multimodal distribution

This example is to show how to form a multimodal distribution from two unimodal ones. We have 3 candidates $a, b, c$. So, the first column in table ?? lists the 6 possible linear orders. The second column shows the frequencies corresponding to profile $p$. Notice that, $a b c$ is the mode at profile $p$. Similarly $b a c$ is the mode at profile $q$ shown in the third column. Now, adding the corresponding frequencies from $p$ and $q$ we have a new profile $p+q$ shown in column 4. $p+q$ has two modes, namely $a b c$ and $b a c$. The same scenario is represented in the above figure ??.

Theorem 5 Let $N_{1}$ and $N_{2}$ be two disjoint non-empty sets of voters, such that $N=N_{1} \cup N_{2}$. For $t \in\{1,2\}$ and let $p^{t}$ be a unimodal profile on $N_{t}$ with mode $R^{t}$. Let $p$ be the superposition of these two unimodal profiles. Let $F$ be a positively discriminating rule from $\mathbb{L}^{N}$ to $\mathbb{W}$.

Then $(x, y) \in F(p)$ and $(y, x) \notin F(p)$ for all $(x, y) \in R^{1} \cap R^{2}$, such that $x \neq y$.

Proof Let $(x, y) \in R^{1} \cap R^{2}$, with $x \neq y$. It is sufficient to prove that $(x, y) \in F(p)$ and $(y, x) \notin F(p)$. Because $R^{1}$ and $R^{2}$ are linear orders it follows that $(y, x) \notin R^{1}$ and $(y, x) \notin R^{2}$. Lemma ?? implies for all $R \in \mathbb{L}_{x y}$ that 


$$
f\left(R, p^{1}\right)>f\left(\tau_{x y} R, p^{1}\right) \text { and } f\left(R, p^{2}\right)>f\left(\tau_{x y} R, p^{2}\right),
$$

Therefore

$$
f(R, p)=f\left(R, p^{1}\right)+f\left(R, p^{2}\right)>f\left(\tau_{x y} R, p^{1}\right)+f\left(\tau_{x y} R, p^{2}\right)=f\left(\tau_{x y} R, p\right) .
$$

Positive discrimination implies that $(x, y) \in F(p)$ and $(y, x) \notin F(p)$.

Remark 3 Theorem ?? shows that in case of two superposed unimodal distributions, $p$, with modi $R^{1}$ and $R^{2}$ the asymmetric part of the outcome, $F(p)$, of a positively discriminating rule, $F$, contains the intersection of these two modi. Because the modi are complete and antisymmetric it follows that

$$
\left(F(p) \triangle R^{1}\right) \cup\left(F(p) \triangle R^{2}\right)=\left(R^{1} \triangle R^{2}\right) .
$$

So, $F(p)$ is on a shortest path between $R^{1}$ and $R^{2}$.

By Lemma ?? this result is also implied if the rule is simultaneously neutral anonymous, monotone and discriminating.

Note that this result generalises to any arbitrary number of superposed unimodal profiles. On the other hand it is not difficult to find superposed unimodal profiles at which the standard problems of Social Choice appear, such as for instance Condorcet cycles.

\section{Conclusion}

One of the main contribution of this paper is in recognizing a common trait in all well-known collective decision rules, that they all choose the mode as the outcome in case the underlying frequency distribution is unimodal. We also try to extend this result outside the domain of unimodal distribution. We present some of the interesting rules like Condorcet consistent rules, Borda rule, Plurality rule retain their property of choosing the mode even if the underlying distribution is $v$-tail perturbed. As Borda rule and Plurality rule are "opposite extremes"in the class of score rules, we expect that a large subclass of score rules also choose the mode at such $v$-tail perturbed unimodal distribution. We also expect that it is possible to predict more about the outcome of multimodal distributions resulting from superposed unimodal distributions.

\section{Appendix}

This appendix is on some combinatorial results used in the proofs above. Throughout this appendix let $\bar{x}$ denote the top ordered candidate at $R^{*}$ and $\underline{y}$ the worst ordered candidate at $R^{*}$. Let $\bar{R}^{*}=\left.R^{*}\right|_{A \backslash\{\bar{x}\}}$ and $\underline{R}^{*}=\left.R^{*}\right|_{A \backslash\{\underline{y}\}}$. In several of the Lemmas below we use recursive formulae based on the cardinalities of $\overline{\mathbb{L}}^{k}=\left\{R: R\right.$ is a linear order on $A \backslash\{\bar{x}\}$ with $\left.d\left(R, \bar{R}^{*}\right)=k\right\}, \overline{\mathbb{L}}_{x y}^{k}=\left\{R \in \overline{\mathbb{L}}^{k}:(x, y) \in R\right\}, \underline{\mathbb{L}}^{k}=\{R: R$ is a linear order on $A \backslash\{\underline{y}\}$ with $\left.d\left(R, \underline{R}^{*}\right)=k\right\}$ and $\underline{\mathbb{L}}_{x y}^{k}=\left\{R \in \underline{\mathbb{L}}^{k}:(x, y) \in R\right\}$, where $x$ and $y$ are different candidates such that in case of the first two sets $\bar{x} \neq x$ and in case of latter two sets $y \neq y$. Let these cardinalities be denoted by $\bar{l}(k), \bar{l}(k, x y), \underline{l}(k)$ and $\underline{l}(k, x y)$ respectively. Further, let $\bar{\delta}=\frac{1}{2}(m-1) \cdot(m-2)$ and $\bar{\rho}=\frac{1}{2} \bar{\delta}$ denote the diameter and radius at $m-1$ candidates.

Because the numbers $l(k), \bar{l}(k)$ and $\underline{l}(k)$ do not depend on the actual naming of the candidates we have that $\underline{l}(k)=\bar{l}(k)$. As the position of the interval $[x, y]_{R^{*}}=\left\{z \in A:(x, z) \in R^{*}\right.$ and $\left.(z, y) \in R^{*}\right\}$ differs in $\bar{R}^{*}$ from that in $\underline{R}^{*}$ some care has to be taken with the numbers $\underline{l}(k, x y)$ and $\bar{l}(k, x y)$. We argue at a later stage that these are equal as well. First we prove the following recursive relations for $l(k)$. 
Lemma 4 For a distance $k$ from $R^{*}$

$$
\begin{aligned}
l(k) & =\sum_{t=0}^{\min \{k, m-1\}} \bar{l}(k-t) \\
& =\sum_{t=0}^{\min \{k, m-1\}} \underline{l}(k-t)
\end{aligned}
$$

Proof Set $\mathbb{L}^{k}$ consists of those linear orders at distance $k-t$ from $\bar{R}^{*}$, where $\bar{x}$ has been swapped $t$ positions downwards. As $\bar{x}$ can at most move over $m-1$ positions the recursive formula follows evidently. The second equality follows similarly by deleting the worst ordered candidate but also because obviously $\bar{l}(k-t)=\underline{l}(k-t)$.

Having this recursive relation on $l(k)$ we can now prove that $l(k)$ strictly increases in $k$ that is for $k$ smaller than or equal to radius distance $\rho$. This completes the proof of Theorem ??.

Lemma $5 l\left(k_{1}\right)<l\left(k_{2}\right)$ for all $k_{1}<k_{2} \leqslant \rho$.

Proof It is obvious that $l(0)=\bar{l}(0)=1$. To prove the lemma, it is sufficient to prove it for the special case that $k_{1}=k-1$ and $k_{2}=k$, where $k \geqslant 1$. Hence we prove that $l(k)-l(k-1)>0$ for all $1 \leqslant k \leqslant \rho$. By the recursive formula of Lemma ?? we have, that if $k \leqslant m-1$, then

$$
l(k)-l(k-1)=\bar{l}(k),
$$

else if $k>m-1$, then

$$
l(k)-l(k-1)=\bar{l}(k)-\bar{l}(k-m),
$$

Therefore in case $k \leqslant m-1$, from Lemma ?? it is obvious that $l(k)-l(k-1)$ is positive. Now consider the case $k>m-1$. As by assumption $\rho \geqslant k$ we have $\frac{1}{4} m(m-1) \geqslant k$. So, $k-m \leqslant \frac{1}{4} m(m-1)-m=\frac{1}{4}\left(m^{2}-\right.$ $m-4 m)<\frac{1}{4}\left(m^{2}-3 m+2\right)=\frac{1}{4}(m-1)(m-2)=\bar{\rho}$. Therefore it is sufficient to prove that $\bar{\rho}$ is closer to $k$ than $k-m$, i.e., $k-\bar{\rho}<\bar{\rho}-k+m$. This is equivalent to $2 k<\bar{\delta}+m$. As $\bar{\delta}+m=\frac{1}{2}(m-1)(m-2)+m=$ $\frac{1}{2}\left(m^{2}-3 m+2+2 m\right)=\frac{1}{2} m(m-1)+1$ the inequality $2 k<\bar{\delta}+m$ is equivalent to $k \leq \rho$. This proves the Lemma.

The following remarks are used later on.

Remark 4 (a) From the proof above we have that $|k-\bar{\rho}|<|k-m+1-\bar{\rho}|$ for all $k \leq \rho$.

(b) Noting that $d\left(R, R^{*}\right)=d\left(-R,-R^{*}\right)$ and $d\left(R^{*}, R\right)+d\left(R,-R^{*}\right)=d\left(R^{*},-R\right)+\bar{d}\left(-R,-R^{*}\right)=\delta$ for arbitrary linear orders $R$, we have that $l(k)=l(\delta-k)$ as well as $l(k, x y)=l(\delta-k, y x)$ for all distances $k$ and different candidates $x$ and $y$.

(c) In particular $(b)$ implies $l(k, x y)=l(\delta-k, y x)$ if $k=\rho$.

We now deduce recursive formula's for $l(k, x y)$.

Lemma 6 Let $x$ and $y$ be candidates.

(a) If $x \neq \bar{x}$, then $l(k, x y)=\sum_{t=0}^{\min \{k, m-1\}} \bar{l}(k-t, x y)$.

(b) If $y \neq \underline{y}$, then $l(k, x y)=\sum_{t=0}^{\min \{k, m-1\}} l(k-t, x y)$. 
Proof (Proof of (a)) Let $x \neq \bar{x}$. Now every linear orders in $\mathbb{L}_{x y}^{k}$ can be thought of as obtained from a linear order at distance $k-t$ from $\bar{R}^{*}$ preferring $x$ strictly to $y$ and then shifting $\bar{x}$ over $t$ position down. By this the formula follows.

(Proof of (b)) Is similar.

Next we show that $l(k, x y)=l(k, a b)$ when cardinalities of the intervals $[x, y]_{R^{*}}$ and $[a, b]_{R^{*}}$ are equal.

Lemma 7 Let $(x, y),(a, b) \in R^{*}$, such that the cardinalities of $[x, y]_{R^{*}}$ and $[a, b]_{R^{*}}$ are equal, say $\kappa$. Then $l(k, x y)=l(k, a b)$.

Proof The proof is by induction on $m \geqslant 3$. The basis $m=3$ is straightforward although a bit cumbersome therefore it is left to the reader. So, here we only deduce the induction step and assume that $m \geqslant 4$.

In case the cardinalities of $[x, y]_{R^{*}}$ and $[a, b]_{R^{*}}$ are $m$, then $x=a$ and $y=b$. So, in this case the equality follows evidently. Therefore suppose that $\kappa<m$. Without loss of generality assume that $(x, a) \in R^{*}$ and $(a, x) \notin R^{*}$.

In case $\kappa=m-1$ we have that $x=\bar{x}, b=\underline{y}$ and $l(k, x y)=\sum_{t=0}^{\min \{k, m-1\}} \bar{l}(k-t, x y)$ and $l(k, a b)=$ $\sum_{t=0}^{\min \{k, m-1\}} \underline{l}(k-t, a b)$. Now $x$ is ordered best at $\underline{R}^{*}$ and $y$ is ordered worst at this order. Where $a$ is ordered best at $\bar{R}^{*}$ and $b$ is ordered worst at that order. Because the cardinalities of $\mathbb{L}^{k}$ and $\mathbb{L}_{x y}^{k}$ do not depend on the actual names of the candidates involved, we have $\bar{l}(k-t, x y)=\underline{l}(k-t, a b)$ for appropriate $k$ and $t$. So, $l(k, x y)=l(k, a b)$.

In case $\kappa \leqslant m-2$ we can find $(c, d) \in R^{*}$ with the cardinalities of $[c, d]_{R^{*}}$ is equal to $\kappa, c \neq \bar{x}$ and $d \neq \underline{y}$. Applying Lemma ?? yields that $l(k, c d)=\sum_{t=0}^{\min \{k, m-1\}} \bar{l}(k-t, c d)=\sum_{t=0}^{\min \{k, m-1\}} \underline{l}(k-t, c d), l(k, x y)=$ $\sum_{t=0}^{\min \{k, m-1\}} \underline{l}(k-t, x y)$ and $l(k, a b)=\sum_{t=0}^{\min \{k, m-1\}} \bar{l}(k-t, a b)$. By the induction hypothesis we have that $\underline{l}(k-t, c d)=\underline{l}(k-t, x y)$ and $\bar{l}(k-t, c d)=\bar{l}(k-t, a b)$ for appropriate $k$ and $t$. So, $l(k, x y)=l(k, c d)=$ $l(k, a b)$.

By Lemma ?? we are able to argue that $\underline{l}(k, x y)=\bar{l}(k, x y)$ as follows.

Lemma 8 For distance numbers $k$ we have $\underline{l}(k, x y)=\bar{l}(k, x y)$.

Proof Note that the cardinalities of $\mathbb{L}^{k}$ and $\mathbb{L}_{x y}^{k}$ do not depend on the actual names of the candidates. So, as shifting the interval $[x, y]_{R^{*}}$ one position up and renaming the alternatives yields the interval $[x, y]_{\bar{R}^{*}}$. Lemma ?? implies that the shifting of the interval has no effect on the number $\underline{l}(k, x y)$ and renaming also has no effect. So, $\underline{l}(k, x y)=\bar{l}(k, x y)$.

To complete the proof of Theorem ?? we prove the following.

Lemma 9 Let distance $k \leq \rho$ and let $x$ and $y$ be different candidates with $(x, y) \in R^{*}$. Then

$$
l(k, x y) \geqslant l(k, y x) .
$$

Proof The proof is by induction on the number of candidates. The basis $m=3$ is straight forward but cumbersome. We leave it to the reader. The induction step where $m \geq 4$ is proved by two cases. In the first case either $x$ is not ordered best at $R^{*}$ or $y$ is not ordered worst at $R^{*}$. Without loss of generality assume that $z$ is ordered worst at $R^{*}$, with $z \neq y$. So, by Lemma ??

$$
l(k, x y)=\sum_{t=0}^{\min \{k, m-1\}} \bar{l}(k-t, x y) .
$$

As $l(k)=\sum_{t=0}^{\min \{k, m-1\}} \bar{l}(k-t)$ and $\bar{l}(s)=\bar{l}(s, x y)+\bar{l}(s, y x)$ for all distances $s$, the induction argument yields that $\bar{l}(s, x y) \geqslant \frac{1}{2} \bar{l}(s)$ for all $s<\bar{\rho}$. Therefore we have $l(k, x y) \geqslant \frac{1}{2} l(k)$. Hence, the desired result 
follows in case $k<\bar{\rho}$. For the case that $k \geqslant \bar{\rho}$ we can find a number $u$ such that $k=u+\lfloor\bar{\rho}\rfloor$. For $0 \leq s \leq u$ by Remark ?? (b) we have $\bar{l}(s+\lfloor\bar{\rho}\rfloor)=\bar{l}(\lceil\bar{\rho}\rceil-s)$ and $\bar{l}(s+\lfloor\bar{\rho}\rfloor, x y)=\bar{l}(\lceil\bar{\rho}\rceil-s, y x)$. This means that $\sum_{t=\lceil\bar{\rho}\rceil-u}^{k} \bar{l}(t, x y)=\frac{1}{2} \sum_{t=\lfloor\bar{\rho}\rfloor+1}^{k} \bar{l}(t)$. Straightforwardly $k<\rho$ and $m \geq 4$ imply $k<\bar{\delta}$. Now Remark ?? (a) and $k<\bar{\delta}$ imply $\lceil\bar{\rho}\rceil-u>\max \{k-m+1,0\}$. Therefore the induction argument yields that $l(k, x y) \geqslant \frac{1}{2} l(k)$ which proves this case.

Next consider the opposite case that is at $R^{*}$ candidate $x$ is ordered best and candidate $y$ is ordered worst. Suppose candidate $z$ is at position ${ }^{\nabla} t$ at $R^{*}$. We can now think of that an ordering in $\mathbb{L}_{x y}^{k}$, with top $z$, is obtained from an ordering at distance $k-t$ from $\left.R^{*}\right|_{A \backslash\{z\}}$, where $z$ has to move $t$ positions to the top. Clearly $t \leq m-2$ as otherwise this operation would result in an order at which $y$ is above $x$. If $z=x$, then this results in $\bar{l}(k)$ orderings. If $z$ is at position 2 , this results in $\widetilde{l}(k-1, x y)$ orders. If $z$ is at position 3 in $R^{*}$ there are $\widetilde{l}(k-2, x y)$ orderings and so on, where $\widetilde{l}(k-t, x y)$ is the number of linear orders at distance $k-t$ from $\left.R^{*}\right|_{A \backslash\{z\}}$. Note that because these numbers are independent of the actual candidate deleted, which means $\widetilde{l}($.$) is the same as \bar{l}($.$) .$

$$
l(k, x y)=\bar{l}(k)+\sum_{t=1}^{\min \{k, m-2\}} \bar{l}(k-t, x y) .
$$

It is sufficient to prove that $2 \cdot l(k, x y)-l(k) \geqslant 0$. This means that we have to prove that $2 \cdot \bar{l}(k)+2$. $\sum_{t=1}^{\min \{k, m-2\}} \bar{l}(k-t, x y)-\sum_{t=0}^{\min \{k, m-1\}} \bar{l}(k-t) \geqslant 0$.

With the induction argument we have that $2 \cdot \sum_{t=1}^{\min \{k, m-2\}} \bar{l}(k-t, x y)-\sum_{t=1}^{\min \{k, m-2\}} \bar{l}(k-t) \geqslant 0$. So, it remains to prove that $2 \cdot \bar{l}(k)-\bar{l}(k)-\bar{l}(k-m+1, x y) \geqslant 0$ or equivalently that $\bar{l}(k) \geqslant \bar{l}(k-m+1, x y)$. As by Remark ?? (a) $k$ is closer to $\bar{\rho}$ than $k-m+1$ we have by Lemma ?? and in case $k \geqslant \bar{\rho}$ using Remark ?? (b) that $\bar{l}(k)>\bar{l}(k-m+1) \geqslant \bar{l}(k-m+1, x y)$ which completes the proof.

The following Lemma completes the proof of Theorem ??.

Lemma 10 Let distance $k \leq \rho$ and let $x$ and $y$ be different candidates with $(x, y) \in R^{*}$. Then

$$
\sum_{z \in A} l(k, x z)-l(k, y z) \geqslant 0
$$

Proof It is sufficient to proof this inequality for the case that $x$ and $y$ are consecutively ordered at $R^{*}$, that is there are no candidate $z$, different from $x$ and $y$, which are ordered between $x$ and $y$ at $R^{*}$. In view of Lemma ?? we have that

$$
\sum_{z \in A} l(k, x z)-\sum_{z \in A} l(k, y z)=l(k, x \underline{y})-l(k, y \bar{x})
$$

Now as $(x, y)$ and $(\bar{x}, y)$ are in $R^{*}$ it follows that by Lemma ?? that $l(k, x \underline{y}) \geqslant \frac{1}{2} l(k) \geqslant l(k, y \bar{x})$. This implies the desired result.

Let $x$ and $y$ be different candidates such that $(x, y) \in R^{*}$. It is sufficient to prove $p l(x)-p l(y)>0$. Let $r_{x}$ and $r_{y}$ denote the rank of $x$ and $y$ in $R^{*}$ respectively. Let $u_{x}=m-r_{x}$ and $u_{y}=m-r_{y}$. Take $\mu_{x y}$ the average of $\bar{\rho}+u_{x}$ and $\bar{\rho}+u_{y}$, so $\mu_{x y}=\bar{\rho}+\frac{1}{2}\left(u_{x}+u_{y}\right)$, where $\bar{\rho}$ is the radius distance of the set of linear orders on $m-1$ candidates. Let $\bar{l}(k)$ be zero if $k$ is not in the interval $[0, \bar{\delta}]$. We need the following lemma to complete the proof of Theorem ??.

Lemma $11 \bar{l}\left(k-u_{y}\right)<\bar{l}\left(k-u_{x}\right)$ for all $k$ such that $u_{x} \leqslant k<\mu_{x y}$.

\footnotetext{
8 There are $t-1$ candidates ordered above $z$ at $R^{*}$
} 
Proof By assumption we have that $k-u_{y}<k-u_{x}$. From $u_{x} \leqslant k$ we have $k-u_{x} \geqslant 0$. From $k<\mu_{x y}$, we have that

$$
\begin{aligned}
k & <\bar{\rho}+\frac{u_{x}+u_{y}}{2} \\
& \Longrightarrow 2 k-\left(u_{x}+u_{y}\right)<\bar{\delta} \\
& \Longrightarrow\left(k-u_{x}\right)-\bar{\rho}<\bar{\rho}-\left(k-u_{y}\right)
\end{aligned}
$$

If $k-u_{x} \leqslant \bar{\rho}$, then the above inequality implies $k-u_{y}<k-u_{x} \leqslant \bar{\rho}$. From Lemma ??, it is obvious that $\bar{l}\left(k-u_{y}\right)<\bar{l}\left(k-u_{x}\right)$. Else if $k-u_{y} \leqslant \bar{\rho}<k-u_{x}$, then the above inequality implies that $\bar{\rho}$ is closer to $k-u_{x}$ than it is to $k-u_{y}$. Applying Lemma ?? and Remark ??, in this case also we have that $\bar{l}\left(k-u_{y}\right)<\bar{l}\left(k-u_{x}\right)$. This completes the proof.

\section{References}

1. Fishburn P., Gehrlein W., An analysis of voting procedures with nonranked voting, Behav Sci 22, 178185 (1977)

2. Gehrlein W., Condorcet winners in dual cultures, Presented at National Meeting of PublicChoice Society, New Orleans, LA (1978)

3. Gehrlein, W., Condorcet's Paradox, Springer, Berlin (2006)

4. Gillett R., Conflict and indecisiveness in collective decision procedures, Presented at Mathematical and Statistical Section of the British Psychological Society, Birmingham, UK (1976)

5. Gillett R., A recursion relation for the probability of the paradox of voting, Journal of Economic Theory 18, 318327 (1978)

6. Grofman B., Regenwetter M., and Tsetlin, I., The impartial culture maximizes the probability of majority cycles, Social Choice and Welfare, 21(3): 387-398 (2003)

7. Grofman B., Marley A.A.J., Regenwetter, M., and Tsetlin I., Behavioral Social Choice: Probabilistic Models, Statistical Inference, and Applications, Cambridge:Cambridge University Press (2006)

8. Merlin, V., Tataru M., Valognes F., On the probability that all decision rules select the same winner, Journal of Mathematical Economics, 33, 183-207 (2000)

9. Riker, W., Liberalism against Populism: A Confrontation between the Theory of Democracy and the Theory of Social Choice, San Francisco: W. H. Freeman \& Co. (1982) 